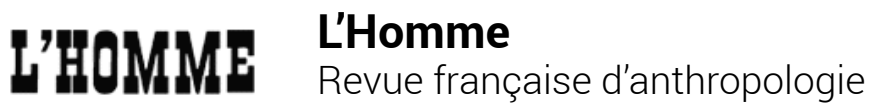

225 | 2018

Varia

\section{Saskia Sassen, Expulsions. Brutalités et complexité dans l'économie globale}

\section{Clara Lecadet}

\section{(2) OpenEdition \\ 1 Journals}

\section{Édition électronique}

URL : http://journals.openedition.org/lhomme/30836

DOI : 10.4000//homme.30836

ISSN : 1953-8103

Éditeur

Éditions de l'EHESS

\section{Édition imprimée}

Date de publication : 15 mars 2018

Pagination : 212-213

ISBN : 978-2-7132-2733-2

ISSN : 0439-4216

Référence électronique

Clara Lecadet, «Saskia Sassen, Expulsions. Brutalités et complexité dans l'économie globale », L'Homme [En ligne], 225 | 2018, mis en ligne le 15 mars 2018, consulté le 08 janvier 2021. URL : http:// journals.openedition.org//homme/30836 ; DOI : https://doi.org/10.4000//homme.30836

Ce document a été généré automatiquement le 8 janvier 2021.

(c) École des hautes études en sciences sociales 


\title{
Saskia Sassen, Expulsions. Brutalités et complexité dans l'économie globale
}

\author{
Clara Lecadet
}

\section{RÉFÉRENCE}

Saskia Sassen, Expulsions. Brutalités et complexité dans l'économie globale. Trad. de l'anglais (États-Unis) par Pierre Guglielmina. Paris, Gallimard, 2016, 371 p., bibl., index, tabl., cartes (« Nrf Essais »).

1 Avec cet ouvrage, la sociologue américaine Saskia Sassen donne à la notion d'expulsion, généralement employée pour désigner les renvois d'étrangers en situation irrégulière ou les expulsions locatives, une portée générique. Les expulsions ont un sens à la fois politique, économique, social et écologique, constitutif d'une «limite systémique » (p. 281). L'auteure situe dans les années 1980 la phase de transition entre un modèle de type keynésien, qui avait permis l'essor à la fois de l'État-providence, des classes moyennes et du syndicalisme ouvrier, et un système marqué par l'essor des inégalités, les hausses vertigineuses du profit des entreprises, les destructions des économies nationales et de la biosphère. À un modèle intégratif, soucieux des individus et de leur protection, succède un système dont la dynamique essentielle est l'expulsion. Non seulement la complexité croissante de nos sociétés n'en a pas aboli la brutalité fondamentale, mais cette complexité nourrit des violences cachées et insaisissables, qui ne sont rendues manifestes que lorsqu'elles menacent l'ensemble du système.

2 Les quatre chapitres illustrent chacun une forme d'expulsion, résultant d'une tendance systémique dont les effets sont souvent invisibles et les manifestations souterraines, à moins de l'observer à son point de rupture et quand elle met l'ensemble du système en crise. En prenant le risque de juxtaposer et de mettre en rapport des réalités qui peuvent sembler hétérogènes au premier abord, Saskia Sassen propose une lecture ambitieuse, globale et transversale des phénomènes qui secouent la planète: contraction des économies nationales, dangers de la financiarisation, déplacements de 
population, destruction de la biosphère. En rassemblant les morceaux d'un puzzle habituellement épars, elle montre le lien causal entre les profondes mutations qui ont lieu dans le Nord et le Sud globaux, attribuant à ce qu'elle nomme des «formations prédatrices ", la responsabilité des phénomènes de paupérisation, d'exclusion et de destruction qui affectent l'ensemble des sociétés au Nord comme au Sud.

Véritable matrice pour penser les diverses formes d'expulsion, ces «formations prédatrices » combinent élites politiques, magnats de l'industrie et de la finance, et capacités systématiques d'appropriation et d'exploitation des ressources. L'accroissement de la richesse du $1 \%$ des plus riches au monde, la croissance des actifs des banques et des revenus des entreprises, le rôle des gouvernements dans la concentration extrême des richesses sont autant d'indices d'un creusement des inégalités sur le plan économique. Cette concentration des richesses se fait au détriment des classes moyennes, paupérisées, et au prix d'une augmentation des chiffres de la pauvreté, même si Saskia Sassen souligne que leurs méthodes de calcul sont variables et que le taux de croissance, par exemple, basé sur l'exclusion de l'économie informelle, laisse de côté des pans entiers de l'économie globale. De même, il convient de réfléchir à la nature des indicateurs de la pauvreté et de l'exclusion sociale dans le Nord et le Sud : «Le chômage, l'émigration, les saisies, la pauvreté et les taux de suicide sont des variables utiles dans les pays du Nord global. Les déplacements de population dus à la guerre, aux maladies et à la famine sont sans doute des variables plus utiles dans le Sud global»(p.79). Il en ressort le constat de "résonances systémiques entre les incarcérations en masse, l'entreposage des réfugiés et les populations déplacées de force» (p. 89, chap. I : «Économies déclinantes, expulsions croissantes »).

Depuis 2006, l'amplification continue du processus d'acquisition des terres par les gouvernements et les entreprises étrangères dans les pays du Sud, pour répondre à une demande croissante en cultures alimentaires et industrielles, fait également partie de ces phénomènes qui ne peuvent se limiter à une seule lecture locale. L'expulsion de nombreux petits propriétaires agricoles et de nombreux villages a en effet été provoquée par la constitution d'un «marché global des terres». Ce processus d'accaparement des terres et de déni des populations locales participe à la fois de la destruction de la biosphère et du déplacement de ces populations vers des bidonvilles. Il suppose l'alignement des États du Sud sur les logiques capitalistes globales, mais il est aussi à l'origine de luttes pour la défense des terres par les habitants qui s'opposent à leur expropriation (chap. II : «Le nouveau marché global des terres »). Le tournant pris par la finance depuis les années 1980 joue un rôle clé dans ces recompositions : son extrême complexification, avec le rôle central des produits dérivés et de la spéculation sur les prêts hypothécaires, est l'une de ces dynamiques souterraines qui ont conduit à des pertes massives, à l'appauvrissement, aux saisies de logements, aux crises récurrentes qui secouent les économies nationales (chap. III : "La finance et ses capacités : la crise comme logique systémique »). Parallèlement, l'industrialisation et l'acquisition de terres ont entraîné la destruction de la biosphère, dans des proportions inégalées (chap. IV : "Terre morte, eau morte »). Les exploitations minières et industrielles n'ont pas seulement rendu des pans entiers de terre infertiles, pollué les nappes phréatiques et les océans, elles ont aussi eu un impact majeur sur la santé des populations. 
5 Saskia Sassen cherche ainsi à penser ensemble la croissance rapide des profits des entreprises, la multiplication des déficits budgétaires des gouvernements, l'intensification des déplacements de populations dans le Sud et l'augmentation des incarcérations dans le Nord, comme autant de manifestations de l'expulsion. La valeur heuristique de ces phénomènes permet d'envisager la dynamique même de nos économies politiques. Si l'usage extensif du terme "expulsion» et des statistiques risque parfois d'en faire perdre de vue les multiples manifestations très concrètes, l'auteure en rappelle le coût humain: pauvreté grandissante, chômage, sans-abris, faim, multiplication des soupes populaires, saisies, augmentation du taux de suicide chez les propriétaires des petits commerces ayant fait faillite. Elle reprend en outre la critique déjà formulée des inégalités croissantes causées par le capitalisme néolibéral, des ravages causés par les programmes de restructuration du FMI et de la Banque mondiale, qualifiés de "régimes disciplinaires" (p.117), sur les services publics des fragiles États africains issus de la période coloniale. Mais elle nuance la vision duale Nord/Sud pour montrer que ces mesures d'austérité, imposées dès les années 1980 aux États pauvres du Sud, trouvent désormais un écho dans l'austérité imposée par ces mêmes organismes en Grèce et dans celle qui sévit en Europe et qui a occasionné un recul fulgurant de ses économies.

6 En filigrane, et alors que le texte est parfois saturé de ces indicateurs, se dessine une réflexion sur la nature des marqueurs de l'économie. Comment mesure-t-on qu'une société est bien portante ? Et peut-être plus important encore, quelles sont ces sphères de la vie sociale que les chiffres oublient, que les statistiques ne peuvent capter et les décideurs encore moins identifier? L'une des idées les plus stimulantes de l'ouvrage est qu'au fur et à mesure que les économies se contractent, se rétrécissent, des espaces informels se développent où les individus s'organisent autrement pour survivre : « Les économies de survie alternatives, qui émergent, existent dans un espace économique différent, à l'extérieur des mesures et des indicateurs formels. Pour l'instant, elles ne sont pas assez nombreuses pour répondre aux besoins des expulsés ou simplement des appauvris» (p. 66). C'est sur cette question ouverte et irrésolue que s'achève le livre : comment identifier et nommer ces espaces créés par l'expulsion? 